\title{
Frontoplastia mini-invasiva
}

\section{Mini-invasive forehead-plasty}

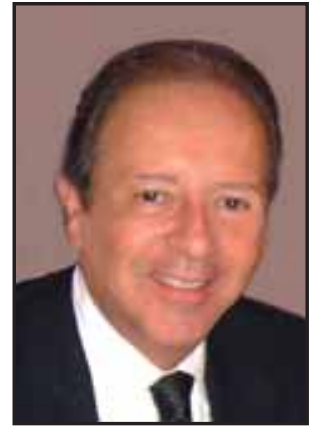

Fuente del Campo, A.
Fuente del Campo, A.*

\section{Resumen}

Una de las causas mas importantes de la flacidez y caída de la piel y de las partes blandas de la cara es la disminución del volumen de su estructura esquelética. Un factor fundamental en la restauración del envejecimiento facial seria el poder recuperar este volumen.

Dado que en la actualidad no se cuenta con un recurso efectivo para recuperar el volumen óseo, lo indicado es restaurar la relación entre las partes blandas y el volumen óseo vigente. Esta es la meta mas importante de la ritidectomía subperióstica. Con una vasta experiencia en este método y buscando minimizar las cicatrices resultantes, hemos desarrollado el enfoque mini-invasivo de este procedimiento de restauración facial. En el presente trabajo describimos la aplicación de nuestro procedimiento para la restauración de la región frontal.

Desde 1998 hemos realizado este procedimiento, con diversas combinaciones y variantes y dependiendo de las necesidades de cada caso, en un total de 521 pacientes: 419 mujeres y 102 hombres de edades comprendidas entre los 45 y los 73 años.

Planteamos las metas y describimos los medios para realizar este procedimiento por vía frontal, transpalpebral y combinando ambas vías, a la vez que damos orientación para lograr los objetivos del procedimiento y evitar complicaciones basándonos en la Anatomía Quirúrgica. Referimos los procedimientos auxiliares que se pueden asociar a este método y describimos los detalles del manejo postoperatorio.

Los resultados obtenidos han sido en general muy satisfactorios tanto para los pacientes como para el cirujano. Mencionamos también las complicaciones observadas.

En conclusión, describimos las observaciones resultantes de nuestra experiencia con este método a lo largo de 10 años, con resultados tan buenos o mejores que con la ritidectomía subperióstica abierta y el porqué es actualmente nuestro procedimiento de elección.

\section{Palabras clave Ritidectomía, Envejecimiento facial}

\section{Código numérico 26-264}

The reduction of the bony structure of the face is the main reason of the flabbiness and fall of the facial soft tissues. Because we do not have already recourses to restore the lost bony volume, a good possibility is to reestablish a good relationship between the soft tissues and the present bony volume. Based on this principle we have developed the procedure of mini-invasive subperiosteal facial restoration. In this paper we described the application of this procedure to restore the frontal region.

Since 1998 we have done this procedure with several variants and combinations according with the needs of each particular case, on 521 patients: 419 females and 102 males, from 45 to 73 years old.

The maneuvers and instrumentation used to perform this procedure through frontal, palpebral or combining both approaches are described. Based on the Surgical Anatomy, orientation and advices to reach the goals of the procedure and to avoid complications are given. The ancillary procedures, which could be associated to this procedure, are described as well as the details of the post-op care.

The observed complications are mentioned and a summary of the results obtained, which have been very satisfactory for the patients and the surgeon, is done.

As a conclusion, the observations from our experience with this method along 10 years and the reasons because this is our first choice procedure, are exposed.
Key words $\quad$ Facelift, Facial ageing

Numeral Code 26-264 


\section{Introducción}

La pérdida de hueso es un factor que acompaña al envejecimiento y se manifiesta entre otras cosas por la perdida de estatura. Esta pérdida de hueso se evidencia claramente en la cara. Los antropólogos han establecido el promedio de reabsorción ósea que ocurre con el envejecimiento en las diferentes edades. Enlow (1) entre muchos otros, concluye en sus estudios que la formación del hueso, que se lleva a cabo en la superficie posterior de los mismos, termina alrededor de los 34 años de edad, mientras que el desgaste óseo de su superficie anterior continúa en gran parte favorecido por la constante actividad muscular.

La disminución tridimensional del volumen de la estructura esquelética de la cara ocasiona flacidez de todos los elementos que se apoyan sobre ella, músculos, tejido celular subcutáneo y piel. La piel pierde elasticidad, la grasa se reabsorbe, los músculos pierden volumen y tono y la fuerza de la gravedad los hace descender.

De esta forma, la pérdida de volumen del hueso frontal ocasiona flacidez de la piel en esta zona, así como el subsecuentemente descenso de las cejas y la blefarochalasia. En la frente es fácil observar la perdida de volumen óseo que cambia progresivamente su contorno. En las mujeres, se aprecia esto con más claridad; la frente convexa de la mujer joven se aplana en el centro y posteriormente se hace cóncava en sentido horizontal, por arriba de los bordes supraorbitarios, siendo más acentuada en la zona del seno frontal.

El hueso esponjoso se pierde rápidamente, mientras que el hueso cortical que constituye los contrafuertes y los marcos orbitarios tarda más en hacerlo, volviéndose éstos progresivamente más evidentes, lo que proporciona evidente dureza a la expresión facial.

Por todo lo anterior, consideramos que la forma adecuada de restaurar el rostro debe considerar necesariamente recuperar el volumen óseo perdido. A falta de un recurso efectivo para lograrlo, lo indicado es restaurar la relación entre las partes blandas y el volumen óseo vigente. Para ello es necesario emplear un procedimiento de planos profundos que permita desplazar y reacomodar las partes blandas que se encuentran sobre el esqueleto. En particular, un procedimiento de vector vertical que permita reubicar las diversas estructuras afectadas por la gravedad en su posición original y no desplazarlas hacia atrás, como ocurría con los procedimientos tradicionales de abordaje preauricular (2-5).

Ideada y desarrollada con este propósito, la ritidectomía subperióstica logra sin duda este objetivo ampliamente. Aunque se consideraba que la ritidecto- mía subperióstica tenía su indicación solo en pacientes con gran deterioro facial, ahora sabemos que lo primero que se deteriora con el envejecimiento es el volumen óseo, posteriormente los tejidos subcutáneos y al final la piel, por lo que consideramos que la ritidectomía subperióstica debe ser el procedimiento básico para cualquier paciente que requiera restauración facial.

Así los pacientes con envejecimiento incipiente se podrán corregir satisfactoriamente con un procedimiento subperióstico aislado de abordaje superior, mientras que los pacientes con mayor deterioro requerirán además un abordaje preauricular y probablemente alguna de las variantes de la ritidectomía subcutánea.

Los pacientes que están entre los 40 y 50 años con ritidosis grados I y II, obtienen excelentes resultados con un procedimiento subperióstico abierto o miniinvasivo sin necesidad de algún otro procedimiento asociado en mejillas o cuello que implique incisiones o cicatrices preauriculares visibles.

La longitud de la incisión, el posible sangrado, la prolongada anestesia postoperatoria de la piel cabelluda y la elevación de la línea de implantación del cabello, son algunos de los argumentos en contra de la vía coronal. Pero todos estos problemas pueden evitarse realizando el mismo procedimiento subperióstico mediante un abordaje reducido, ya sea endoscópico (6-10) o mini-invasivo (11-14).

La ritidectomía subperióstica endoscópica y la mini-invasiva, se basan en los mismos principios de la subperióstica abierta, con las diferencias inherentes a emplear incisiones pequeñas, requerir menos hemostasia y menos suturas, ahorrando tiempo quirúrgico y anestesia para el paciente. Por supuesto que esto ocurre cuando ya se ha superado la curva de aprendizaje; al principio resulta más difícil, ya que es necesario acostumbrarse en el caso de la endoscopia, a trabajar con una imagen bidimensional y en un campo diferido, es decir con las manos sobre el paciente y los ojos en el monitor y en el caso de la mini-invasiva, a trabajar a través de pequeñas incisiones.

Dada la amplitud del tema, en este trabajo solo nos referiremos al procedimiento que empleamos actualmente para restaurar el envejecimiento de la región frontal, al que llamamos frontoplastia mini-invasiva y con el cual hemos podido obtener consistentemente buenos resultados, reduciendo el periodo de recuperación de los pacientes.

\section{Material y método}

Desde 1995 realizamos diversas variantes de ritidoplastia mini-invasiva, pero desde 1998 estamos reali- 


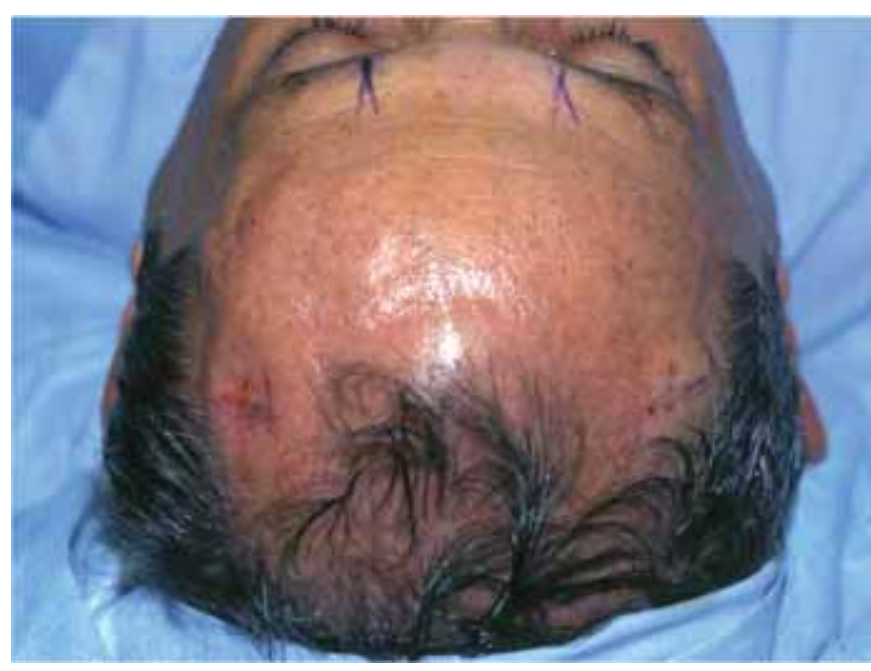

Fig. 1. Planteamiento quirúrgico del abordaje mini-invasivo de la región frontal, ubicación de las incisiones, trayecto de los nervios supraorbitarios y músculos a tratar.

zando este procedimiento, producto de nuestras experiencias y que describiremos a continuación.

Con algunas combinaciones y variantes, dependiendo de las necesidades de cada caso, hemos realizado la técnica en un total de 521 pacientes: 419 mujeres y 102 hombres, de edades comprendidas entre los 45 y los 73 años.

El paciente debe ser valorado en reposo, cuidando que no accione el músculo fronto-occipital y mirando al frente en posición ortofórica. En la mayoría de los casos los pliegues horizontales de la frente se producen por la contracción crónica del músculo frontooccipital que la persona acciona por costumbre para enfatizar alguna expresión o en forma refleja para elevar las cejas y aliviar el peso que representa la piel redundante sobre los párpados superiores. Con esta maniobra se puede apreciar el descenso que han sufrido las cejas y la verdadera cantidad de piel redundante en el párpado superior, habitualmente diferente a la que se aprecia de primera intención.

Para fines prácticos, calificamos el grado de envejecimiento facial en cuatro grados:

Grado I: Flacidez y descenso incipiente de la cara.

Grado II: Flacidez y descenso evidente de la cara e incipiente flacidez del cuello.

Grado III: Incipiente redundancia de la cara y evidente flacidez del cuello.

Grado IV: Evidente redundancia de la cara y del cuello.

Indicaremos diversas variaciones de nuestro procedimiento quirúrgico dependiendo del grado de envejecimiento del paciente y de sus características faciales.

\section{Procedimiento Quirúrgico}

La técnica quirúrgica puede llevarse a cabo bajo anestesia local o general; en el primer caso se efectúa el bloqueo bilateral de los nervios supraorbitario e infraorbitario. En ambos casos una vez preparada el área quirúrgica, se realiza la infiltración de una solución vasoconstrictora consistente en $1 \mathrm{ml}$ de Epinefrina al 1:100.000 y $10 \mathrm{ml}$ de Marcaína al 5\% en $150 \mathrm{ml}$ de solución tipo Hartman. Para los casos bajo anestesia local agregamos $20 \mathrm{ml}$ de Lidocaína al 2\%. El procedimiento lo realizamos siempre siguiendo una secuencia de arriba hacia abajo (Fig. 1).

Al restaurar la región frontociliar, tenemos como propósitos darle altura y contorno estético a las cejas, corregir o mejorar las líneas de expresión de la frente y balancear la función de los músculos que participan.

Las vías de acceso que empleamos para realizar este procedimiento son: una incisión de $2 \mathrm{~cm}$. de longitud por arriba de la mitad lateral de la ceja, dentro de la línea de implantación del cabello y realizada bilateralmente. Para ubicar esta incisión, se trazan dos líneas que partiendo del margen externo del ala nasal se dirigen diagonalmente hacia arriba y hacia afuera hasta la línea de implantación del cabello, pasando una de ellas por el centro del iris y la otra por el borde lateral de la córnea (Fig. 2). La incisión se traza entre estas dos líneas, discretamente por dentro y a lo largo de la línea implantación del cabello (15).

En muchos casos, también se hace necesaria una incisión en el párpado superior del tipo de la empleada para una blefaroplastia, a lo largo del pliegue supratarsal (10 a $12 \mathrm{~mm}$ del borde ciliar en su porción central), siguiendo el trazo de una blefaroplastia convencional.

Los casos que tienen poca redundancia de piel en párpado superior (ritidosis I), habitualmente se corrigen elevando moderadamente la ceja por la vía superior, lo que hace innecesaria la incisión palpebral. Es importante diferenciar si la redundancia del párpado es de piel o del músculo orbicular. Los pacientes con obvia redundancia cutánea, hipertrofia del músculo orbicular superior o bolsas grasas evidentes, requieren abordaje transpalpebral.

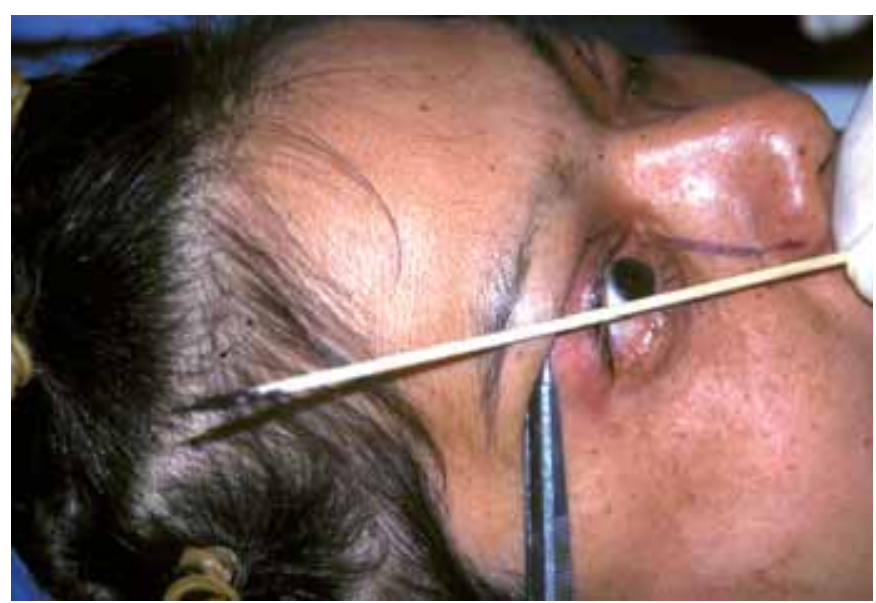

Fig. 2. Planteamiento y trazado de la incisión frontal. 

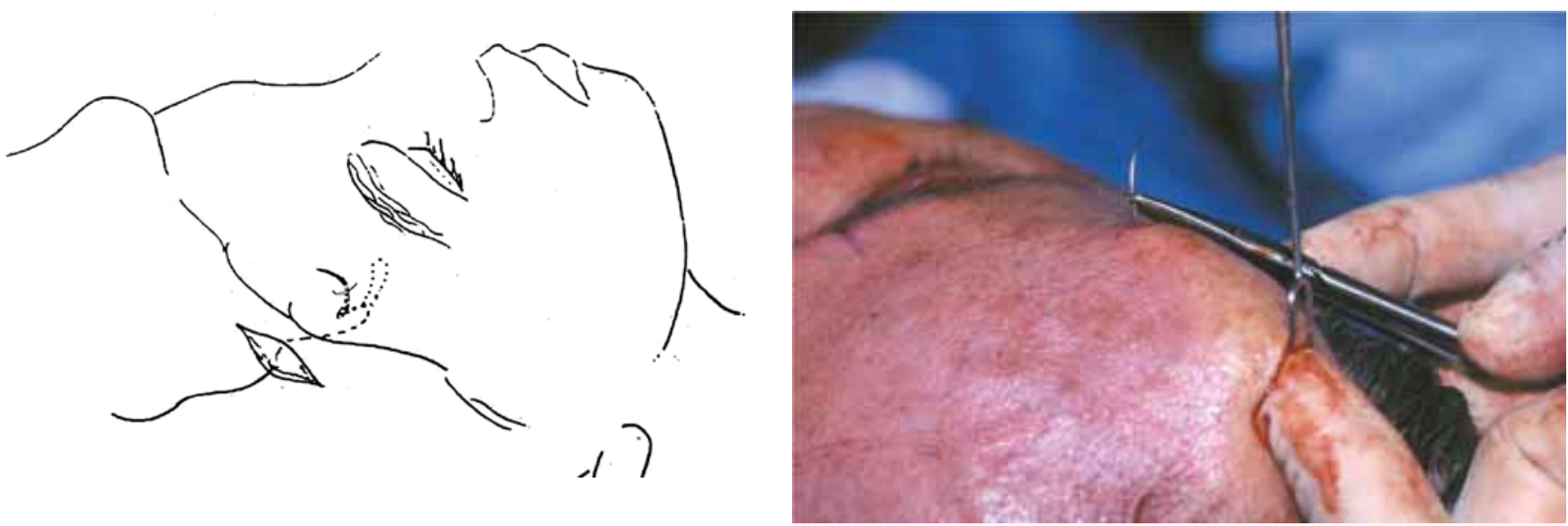

Fig. 3. Sutura de "ida y vuelta" para elevar las cejas. a) Esquema del procedimiento. b) Control "digital” para la extracción de la aguja.

La disección subperióstica de la región frontal representa un plano limpio, que nos permite elevar las cejas o tratar los músculos de la región fronto-orbitaria, ya sea por la vía superior descrita, a través de la vía transpalpebral o combinando ambas.

\section{Procedimiento transfrontal}

Se diseca en el plano subperióstico exclusivamente la zona del hueso frontal sobre la que se inserta el músculo fronto-occipital,. No es necesario despegar hacia la región fronto-parietal, donde se encuentra la fascia fronto-occipital ya que es un elemento deslizante, no adherido al hueso. De hecho en la mayoría de las ocasiones no disecamos por arriba de la incisión frontal para evitar ampliar la dimensión vertical de la frente. Una vez hecho el despegamiento se incide horizontalmente el periostio y la fascia posterior del músculo en su porción frontal (una o varias veces), $3 \mathrm{~cm}$ por arriba de las cejas, desde la cresta temporal de un lado hasta la contralateral. De esta forma las cejas, que son una estructura cutánea, podrán ser desplazadas libremente hacia arriba.

La ubicación de las cejas depende en gran parte del balance muscular entre los músculos elevadores y depresores. Mientras que el músculo fronto-occipital es el único músculo elevador, tenemos varios depresores: el orbicular que actúa sobre toda la ceja, el procerus y depresor ciliar que descienden la cabeza de la ceja y el corrugador que la medializa pero también la desciende un poco, aunque fundamentalmente la medializa. La relajación de los músculos depresores suaviza la expresión de la cara y libera al músculo fronto-occipital de sus antagonistas, favoreciendo la elevación de las cejas. Sin embargo, el propósito no es solo elevar la ceja a una altura determinada, sino darle el contorno adecuado y las proporciones estéticas acordes con la dimensión vertical del párpado y el surco supratarsal. La escisión parcial o debilitamiento de los músculos procerus y depresor ciliar, está indi- cada sólo en aquellos casos en que es necesario elevar el tercio interno de las cejas y deberá realizarse solo cuando sea posible reubicar su tercio externo por arriba del nivel del tercio medial. En la actualidad, se considera que la expresión armónica de la cara se relaciona con cejas discretamente diagonales cuyo tercio lateral se encuentre por arriba de su tercio medial. Potenciar la acción del músculo fronto-occipital compromete a elevar proporcionalmente el tercio externo de las cejas, de otra manera obtendremos cejas diagonales de arriba hacia abajo y de adentro hacia afuera que dan a la cara expresión de sorpresa o de tristeza, debido a que el músculo fronto-occipital actúa elevando solo la porción medial de la ceja y en muy pocos casos tiene algún efecto sobre su porción lateral. Para conservar la elevación de las cejas, es necesario fijarlas selectiva e intencionadamente al nivel deseado. Para tratar los músculos depresores la vía transpalpebral superior es la indicada.

Es fundamental la hemostasia de la zona; una vez verificada, se hace la suspensión del tercio lateral de las cejas mediante puntos de "ida y vuelta" (Fig. 3). A través de la incisión frontal superior, se eleva el colgajo frontal empleando un retractor y se introduce la aguja de sutura (empleamos sutura reabsorbible a largo plazo 4-0), para atravesar la piel $2 \mathrm{~cm}$ por arriba de la ceja. Pasamos la aguja casi en su totalidad y regresamos en un plano subcutáneo a lo largo de $1 \mathrm{~cm}$, para después volver al espacio subperióstico extrayéndola por la misma incisión frontal superior. Esta sutura llamada de "ida y vuelta" permite tomar una "mordida" gruesa de los elementos subcutáneos y traccionar la ceja sin dejar puntos externos que haya necesidad de retirar o que puedan marcar la piel. Se traccionan las dos puntas de la sutura hasta llevar la ceja a la altura deseada y se anudan, anclando al periostio y a la dermis del borde superior de la incisión (16).

Si se prefiere, se pueden anclar a un túnel cortical en el hueso frontal (Fig .4); esto se hace con una broca 


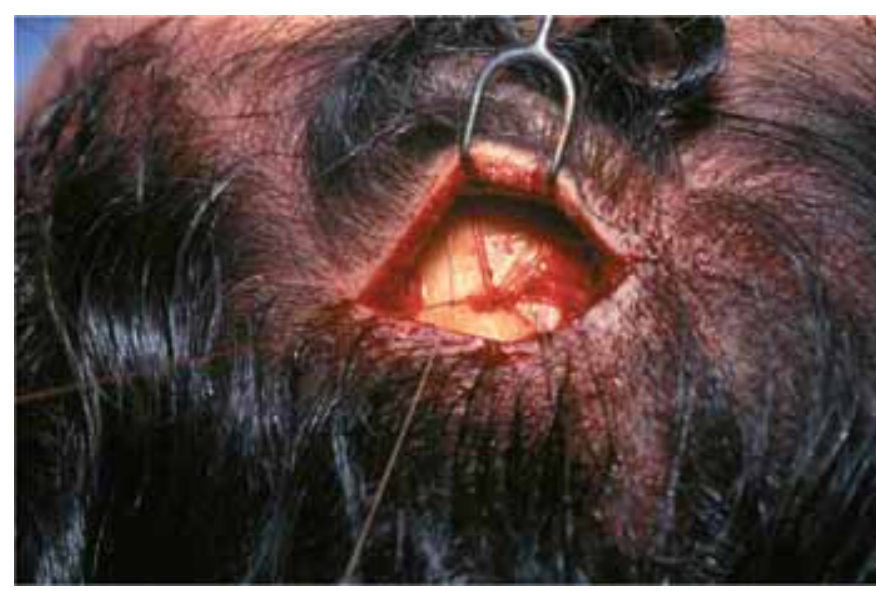

Fig. 4. Anclaje de las suturas al túnel monocortical realizado en el hueso frontal.

de $2 \mathrm{~mm}$ introducida diagonalmente hasta observar un pequeño sangrado que indica que hemos llegado a la esponjosa. Enseguida se hace una perforación convergente, calculando que ambas perforaciones se conecten en su extremo interno para establecer un túnel. Debemos calcular que la separación entre ambas perforaciones debe permitir el libre paso de la aguja curva que tienen las suturas atraumáticas. Se pasa la aguja a través del túnel cortical en el hueso frontal y se tensa hasta llevar la ceja a la altura deseada. Se colocan dos o tres puntos similares distribuidos en la zona para asegurar la suspensión (Fig. 5).

Tenemos que calcular de acuerdo con cada caso el grado de tracción aplicado a las cejas; cuando las cejas se encuentran en un nivel asimétrico, es necesario corregirlas aplicando diferente grado de tracción en ambos lados. En algunos casos la elevación de las cejas ocasiona que se aglomere piel a nivel de la incisión frontal, piel que debemos resecar mediante una incisión perpendicular realizada en el centro del borde inferior de la incisión frontal, que delimita la piel sobrante, en forma de dos pequeños triángulos. Las heridas se cierran con sutura intradérmica, empleando el mismo material reabsorbible.

\section{Procedimiento transpalpebral}

$\mathrm{Al}$ igual que en una blefaroplastia convencional, se reseca piel y músculo orbicular redundante. Habitualmente es conveniente resecar más músculo que piel para eliminar el volumen redundante, pero teniendo en cuenta la piel necesaria para cubrir adecuadamente el caprichoso contorno de esta zona en que se alternan concavidad y convexidad. Se diseca con tijera en forma roma entre el septum orbitario y el músculo orbicular (cuidando no abrir los depósitos de grasa palpebral para evitar su extrusión), hasta llegar al reborde del techo orbitario donde se incide horizontalmente el periostio. Por esta vía y teniendo en cuenta la ubicación de los nervios supraorbitario y trocle-

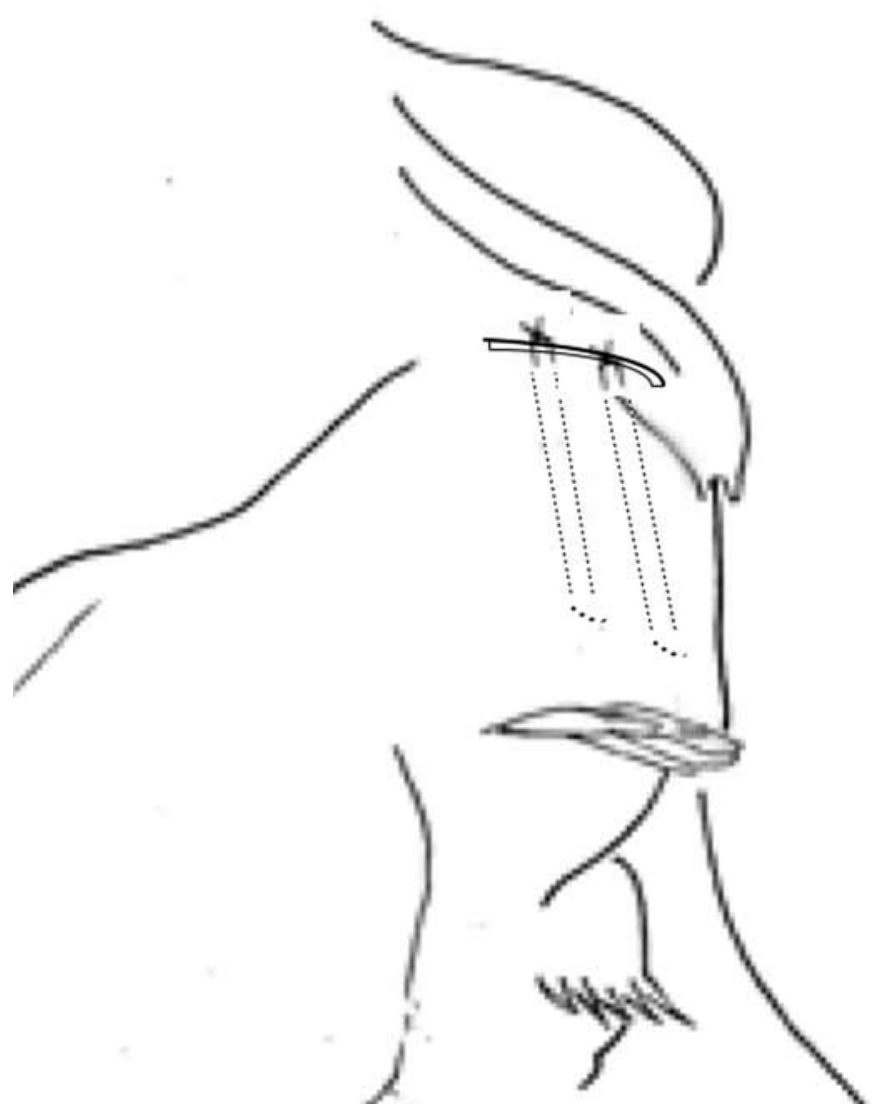

Fig. 5. Ubicación de las suturas para la elevación de las cejas.

ar, se realiza la disección subperióstica de la región frontal empleando una legra larga maleable y enseguida, mediante una legra de filo o una tijera, se hacen cortes horizontales en el periostio y en la fascia posterior del músculo fronto-occipital, de una cresta temporal a la contralateral (solo cuando no se ha hecho previamente por la vía frontal).

El músculo orbicular es debilitado y disociado del músculo frontal mediante la resección de una tira a nivel del arcus marginalis, que no es otra cosa que la inserción del músculo orbicular sobre el periostio. En algunos casos es necesario seccionar transversalmente las fibras del músculo orbicular en uno o varios sitios, pero siempre lateralmente al nervio supraorbitario.

La disección roma por detrás del músculo orbicular, en el ángulo superointerno de la órbita, permite localizar el músculo depresor de la ceja, cuyas fibras corren en sentido casi vertical y se caracterizan por su color rojo vinoso. Disecando hacia arriba se localiza la porción medial del corrugador y se secciona por dentro del nervio supraorbitario, justo antes de su inserción medial, cuidando no desfuncionalizarlo totalmente para evitar que las cejas se separen demasiado y den un aspecto hipertelórico, tal y como se puede comprobar en los pacientes en que se paralizan estos músculos mediante toxina botulínica. Continuando hacia la zona glabelar, la disección se hace en dos planos, uno subcutáneo y otro subperióstico, que- 

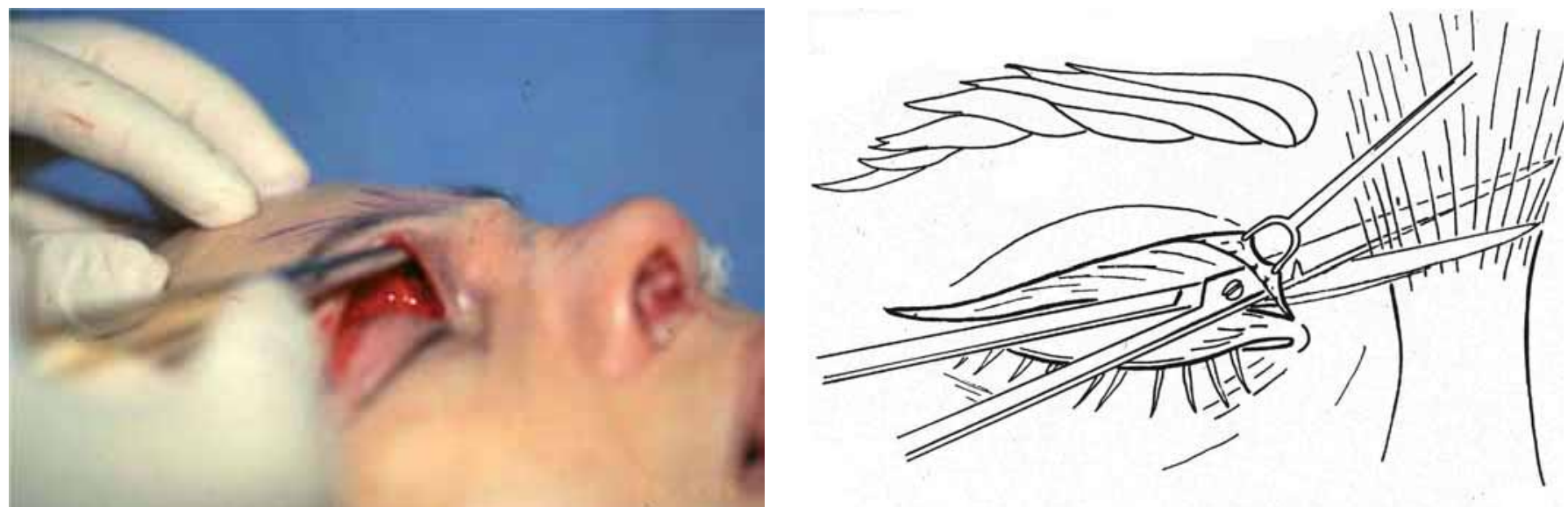

Fig. 6. Disección y ablación del músculo procerus. a) Disección del túnel subcutáneo. b) Esquema del procedimiento de ablación.

dando entre ambos el músculo procerus. Este, al igual que los diversos músculos depresores, son seccionados con tijera a diferentes niveles para desvitalizarlos y desfuncionalizarlos (Fig. 6); esta maniobra que produce un poco de sangrado que se controla mediante compresión local durante unos minutos. No es recomendable resecar fragmentos de músculo, ya que esto puede ocasionar depresiones visibles.

Dependiendo de las características del paciente, se decide qué músculos es necesario tratar.

La tracción vertical ejercida para elevar la ceja, así como la mejoría de la función elevadora del músculo fronto-occipital, habitualmente reduce de forma notable la presencia de las bolsas grasas palpebrales superiores; sin embargo, cuando persisten evidentes, se toma su cápsula con una pinza fina y se electrofulguran, lo que hace que se retraiga la cápsula llevando la bolsa hasta su lugar de origen. En los casos con bolsas palpebrales más severas se colocan puntos de plicatura en la cápsula de la bolsa para lograr su intrusión.

Cerramos la incisión palpebral mediante sutura intradérmica (nylon 5-0), tomando la piel del borde superior y la piel del borde inferior junto con el tarso en el borde inferior para acentuar la definición del surco supratarsal.

\section{Manejo postoperatorio}

Los extremos de las suturas se fijan con pequeños parches de papel microporo y cubrimos todas las incisiones con tiras del mismo material; colocamos tiras horizontales en la frente hasta cubrirla con dos capas. La intención de este recubrimiento con microporo es contener la distensión que sufren los tejidos por el edema y evitar que el proceso inflamatorio pudiera desprender algunas de las suturas colocadas en el interior, perdiéndose parte de la sujección realizada. Igualmente, al contener el edema, la presión se ejerce sobre el lecho quirúrgico reduciendo las posibilidades zonas donde colocamos papel microporo, prácticamente no presentan equímosis.

Cuando consideramos la posibilidad de que ocurra sangrado, dejamos un drenaje activo, tipo Minivac ${ }^{\circledR}$ y protegemos la zona con gasas, apósitos y una venda alrededor de la cabeza con mínima presión, capaz de sostener el material de cura pero sin apretar. Al resto de los pacientes los dejamos descubiertos, sin vendaje alguno e indicamos la aplicación constante de compresas frías para favorecer la vasoconstricción de la zona.

Como la sutura de las heridas se realiza con material reabsorbible y sutura intradérmica, hacia el $4^{\circ}$ o $5^{\circ}$ día eliminamos los nudos y cubrimos las heridas con tiras delgadas de microporo: el resto de las suturas se dejan. El papel microporo de la frente lo retiramos a los 8 días. Volvemos a ver al paciente 15 a 20 días después, cuando el edema prácticamente ha desaparecido y extraemos los fragmentos de sutura que pudieran haberse hecho visibles.

Este método es particularmente conveniente con los pacientes foráneos o extranjeros y para los pacientes cuyas actividades les limitan la posibilidad de asistir al consultorio con frecuencia para retirar las suturas.

\section{Procedimientos auxiliares}

En muchos casos realizamos la frontoplastia miniinvasiva asociada a algún tipo de ritidectomía o ritidoplastia. Sin embargo con frecuencia también la realizamos como procedimiento aislado, asociado a blefaroplastia o a ritidoplastia centrofacial (17-19). En los pacientes con ptosis palpebral senil hacemos su corrección en el mismo tiempo quirúrgico, mediante la plicatura de la aponeurosis del músculo elevador a nivel de su inserción sobre el tarso. En algunos casos aplicamos lipoinyección para redondear y suavizar el contorno de la frente.

El tratamiento de la piel mediante dermoabrasión, peeling láser o peeling químico, así como la corrección de cicatrices previas, lo asociamos a la colocación de injertos de cabello en zonas alopécicas, parti- 

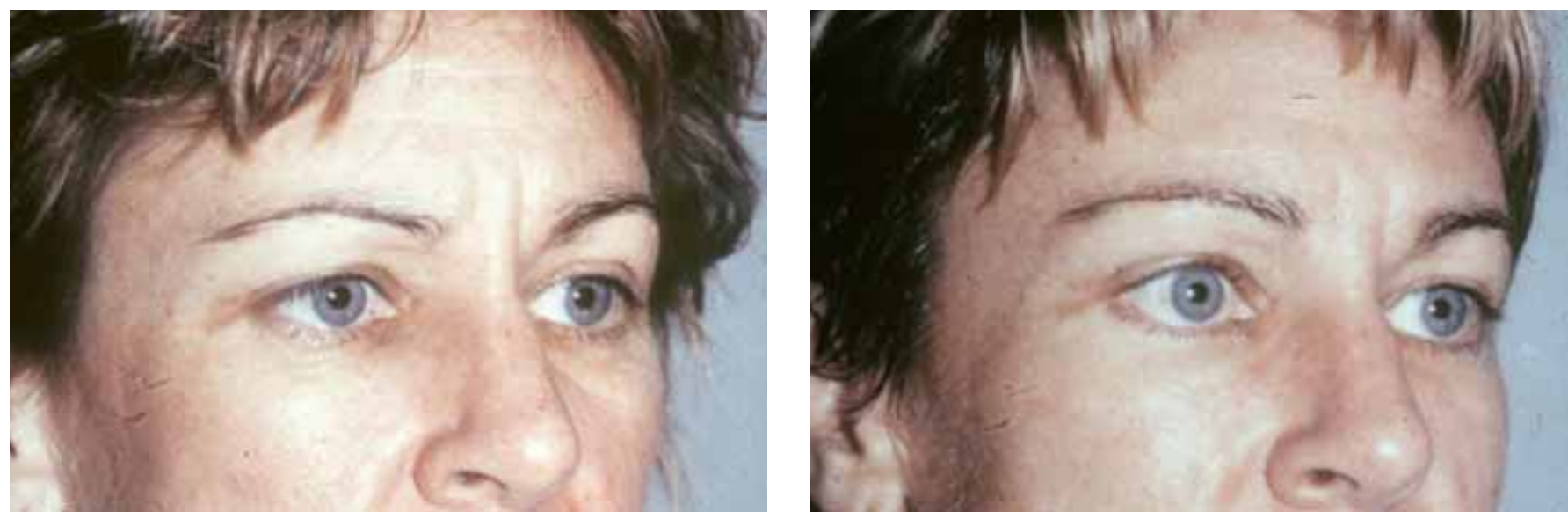

Fig. 7. a) Mujer de 48 años con ritidosis grado II. b) Aspecto postoperatorio un año después de su restauración frontal mini-invasiva por vía frontal, sin abordaje palpebral.

cularmente en pacientes con secuelas de ritidectomía, para corregir la línea de implantación del cabello o reconstruir la patilla.

La frontoplastia mini-invasiva también es un procedimiento muy seguro que puede ser asociado a otros procedimientos quirúrgicos faciales tales como rinoplastia, otoplastia, etc.

\section{Resultados}

Hemos realizado procedimientos mini-invasivos de restauración facial durante 17 años; en el transcurso de este tiempo hemos hecho diversas modificaciones al procedimiento. Desde 1998 estamos realizando básicamente el procedimiento que es tema de este trabajo, aplicándolo con algunas variantes y diversas combinaciones dependiendo de las necesidades de cada caso, para un total de 521 pacientes: 419 mujeres y 102 hombres (Fig. 7).

La cicatriz de las incisiones frontales desaparece como mucho en 3 meses, confundiéndose con la línea de implantación del cabello. Las complicaciones que hemos observado son: hipoestesia de la región frontal (14\% de los casos), aunque menos evidente y de

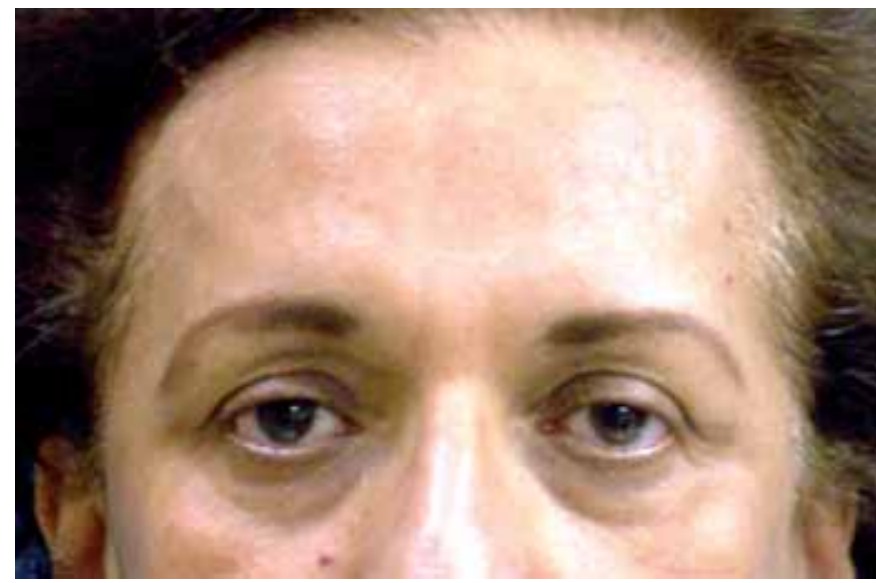

menor duración que con el abordaje coronal; paresia de la rama temporal del facial (3\%), por periodos que oscilaron de una semana a mes y medio, recuperándose totalmente en forma espontánea; hematoma en el $8 \%$ de los casos, todos ellos de proporciones menores, que fueron resueltos con procedimientos simples en el área de recuperación o en el consultorio.

El endoscopio es un instrumento muy útil que permite la magnificación de la imagen en el monitor así como observar con gran detalle el área quirúrgica, por lo que es conveniente contar siempre con uno para el caso de necesitar verificar algún detalle, como el ver si se está en el plano quirúrgico adecuado, si trabajamos en la estructura deseada, si hay algún vaso sangrando, etc. Permite también trabajar con seguridad en las zonas de riesgo, mientras nos familiarizamos con el método mini-invasivo sin endoscopio (Fig. 8).

\section{Conclusiones}

El vector vertical de este procedimiento permite restaurar la frente en forma natural y armónica, respetando la dimensión vertical de la frente o ampliándola cuando se considera conveniente.

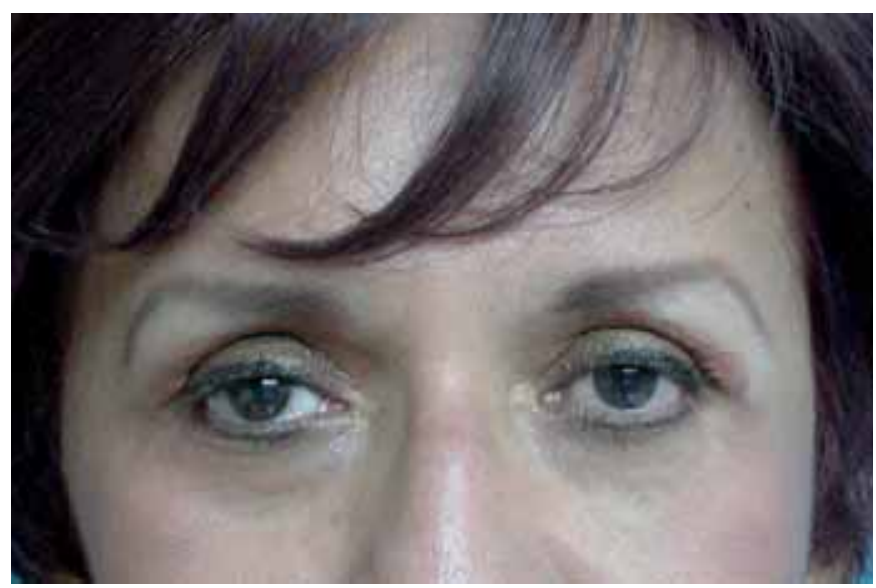

Fig. 8. a) Mujer de 52 años con ritidosis grado III. b) Aspecto postoperatorio 17 meses después de su restauración frontal mini-invasiva por abordaje mixto, asociada a blefaroplastia e injertos de cabello. 

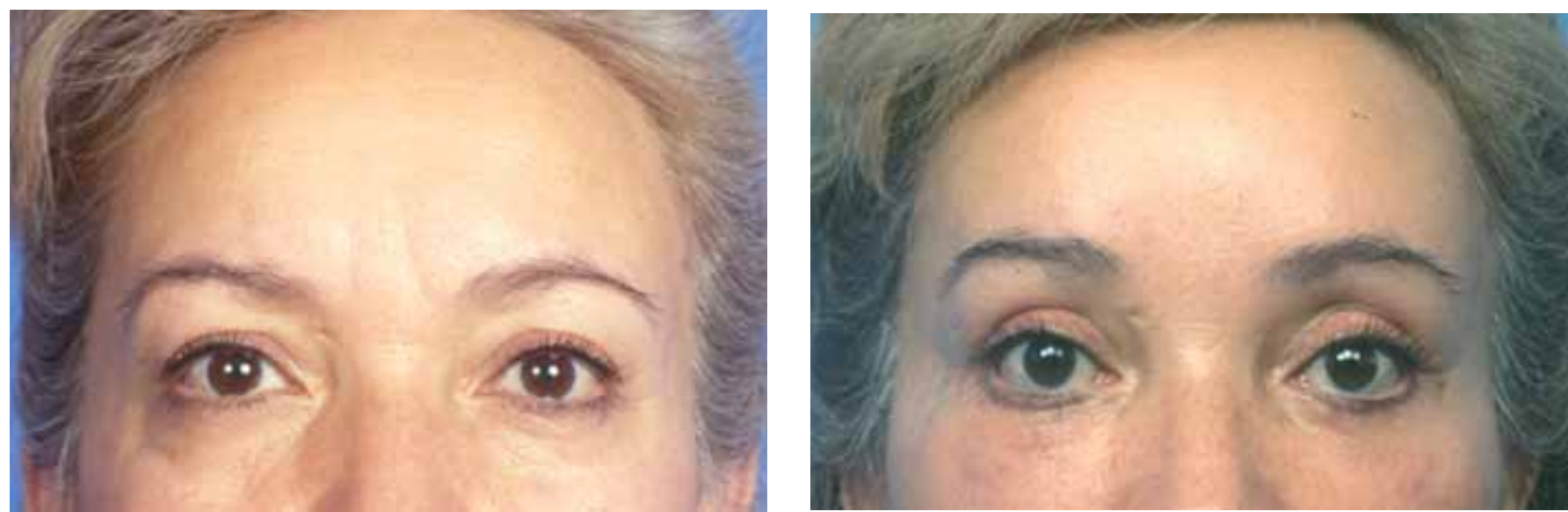

Fig. 9. a) Mujer de 55 años con ritidosis grado IV. b) Aspecto postoperatorio 10 meses después de su restauración frontal mini-invasiva por abordaje mixto, asociada a blefaroplastia y lipoinyección frontal.

Permite tratar en forma específica la piel y cada uno de los músculos de la expresión que participan en la zona. Prácticamente se obtienen los mismos resultados que con el abordaje coronal, pero sin el estigma de la cicatriz, la perdida de cabello y los prolongados periodos de hipoestesia. Por tratarse de incisiones pequeñas se reduce el sangrado, causa menos edema y permite conservar el drenaje venoso y linfático, acelerando el proceso de desinflamación.

Las incisiones frontal y transpalpebral permiten acceso a todas las estructuras de la zona.

Para el cirujano que está familiarizado con la técnica subperióstica, el procedimiento resulta más fácil, rápido y eficiente que la ritidectomía subcutánea tradicional. Las ventajas de este procedimiento incluyen un plano de disección prácticamente avascular, lejos de los nervios y que permite desplazar los tejidos blandos de la frente en una sola capa continua o colgajo, sin alterar las relaciones anatómicas entre la piel y los tejidos blandos profundos. Es un colgajo con excelente vascularización que permite realizar al mismo tiempo y con seguridad otros procedimientos agregados. Conserva la relación anatómica entre el periostio, los músculos y la piel, así como el efecto "amortiguador" de la tracción, que permite que ésta se haga sobre el periostio (una vez liberado) logrando indirectamente tensar la piel en forma suave y natural Además respeta el aporte sanguíneo subcutáneo de la piel, por lo que no la desvitaliza (Fig. 9).

La frontoplastia mini-invasiva ha demostrado su eficiencia independientemente del grado de deterioro o ritidosis del paciente. Es simple, fácil de realizar y de efecto muy evidente, logrando resultados de mayor duración y con un alto grado de satisfacción para los pacientes. Sólo es necesario familiarizarse con la anatomía de la zona y un poco de práctica, para lograr los excelentes resultados que todos deseamos.

\section{Dirección del autor}

Dr. Antonio Fuente del Campo

CAP Interlomas \#26, México, DF. 52786, México. e-mail: afuentedelcampo@prodigy.net.mx

\section{Bibliografía}

1. Enlow DH.: "Manual sobre crecimiento facial". Ed. Intermedica, Buenos Aires 1982.

2. Fuente del Campo A.: "Facelift without preauricular scars". Plast Reconstr Surg .1993; 92: 642

3. Fuente del Campo A. "Subperiosteal face-lift with staged suspension: a facial restoration method without preauricular scars". In: Psillakis J, ed. Deep-lifting techniques. New York: Thieme Medical, 1994, Pp: 76-89.

4. Fuente del Campo A.: "Technique and auxiliary maneuvers for a face-lift without preauricular scars". Oper Tech Plast Reconstr Surg 1995;2:116.

5. Ramirez OM, Maillard GF, Musolas A: "The extended subperiosteal facelift: a definitive soft-tissue remodeling for facial rejuvenation". Plast Reconstr Surg 1991; 88:227.

6. Isse N.: "Endoscopic facial rejuvenation: endoforehead, the functional lift". Aesthetic Plast Surg 1994;18:21.

7. Ramirez OM: "Endoscopic subperiosteal browlift and facelift". Clin Plast Surg 1995, 22:639.

8. Fuente del Campo A.: "Ritidectomía Subperióstica Endoscópica". Cir.plást. iberolatinoam. 1994, 20: 393.

9. Fuente del Campo A.: "Facial rejuvenation (endoscopic): technique and rationale". In: Fodor PB, Isse NG, eds. Endocopically assisted aesthetic plastic surgery. St. Louis: Mosby, 1996, Pp: 63-77.

10. Fuente del Campo A.: "The endo-facelift, basic and options". Rev Clin Plast Surg 1997;24:309.

11. Fuente del Campo A.: "Mini-invasive facial rejuvenation without endoscopy". Aesthetic Surg J. 1996;16(2):129.

12. Fuente del Campo AB, Gordon C, Kiesler Bergman O.: "Evolution from endoscopic to mini-invasive facelift: a logical progression". Aesthetic Plast Surg 1998; 22:267.

13. Knize DM: "Limited incision forehead lift for eyebrow elevation to enhance upper blepharoplasty". Plast Reconstr Surg 1990; 86:682.

14. Knize DM.: "Transpalpebral approach to the corrugator supercilli and procerus muscles". Plast Reconstr Surg 1995;95:52.

15. Fuente del Campo A, Greensmith A: "Minimally invasive approach to facial rejuvenation". In: Aesthetic Surgery for the Face. Ed. Peled-Manders. Taylor and Francis, London, 2004, Pp:1-28.

16. Fuente del Campo A.: "Update on Minimally Invasive facelift technique". Aesthetic Surgery Journal, 2008; 28:51.

17. Fuente del Campo A.: "Centrofacial lifting". Perspect Plast Surg 1993;7:87.

18. Fuente del Campo A.: "The subperiosteal rhytidectomy and the lower lid incision approach to the nasolabial fold". In: Bernard BW, ed. Surgical restoration of the face. Butterworth-Heinemann, 1996, Pp:169-94.

19. Fuente del Campo,A. "Ritidectomía Mini-invasiva". Vol. II, Cirugía Plástica, Reconstructiva y Estética. Ed. Coiffman F., Salvat, Barcelona. 2007, Pp:1131-1146. 


\title{
Comentario al trabajo ufrontoplastia mini-invasiva»
}

\author{
Dr. Guillermo Vázquez \\ Sociedad Argentina Cirugía Plástica. Director del Capítulo de Estética FILACP 2006-2008.Director de Eventos Interna- \\ cionales FILACP 2008-2010
}

Es muy grato comentar un trabajo como el que nos presenta el Dr. Antonio Fuente del Campo, ya que uno puede apreciar en el mismo su vasta experiencia en la Cirugía Craneo-Facial y en donde aplica el concepto tridimensional de las estructuras en la restauración del volumen entre los tejidos blandos y óseos. En la introducción, el autor realiza una descripción anatómica y fisiológica del envejecimiento facial que es prácticamente perfecta para poder comprender la táctica para la restauración.

Muchos autores se han preocupado por solucionar la ptosis de la ceja (Onur, Turowski, etc.) y han descrito varias formas de suspensión, algunas de las cuales no obtenían el resultado deseado a largo plazo debido a que había una recidiva de la ptosis después del año. Deteniéndonos en la parte netamente técnica, el Dr. Fuente del Campo nos brinda un aspecto importante de recalcar ya que evita la recidiva, como es la apertura del periostio y la fascia posterior del músculo frontal, porque de esa forma permite que la ceja se movili- ce y que se desplace conjuntamente con las partes blandas hacia arriba; si no se realiza esta apertura, el ascenso de la ceja sería muy imperceptible con esta pequeña incisión de piel.

En el procedimiento transparpebral se necesita evaluar muy bien la acción de los músculos agonistas y antagonistas, ya que de eso dependerá el tratamiento a seguir. En nuestras manos hemos tenido algunos edemas en la región glabelar que han perdurado hasta un mes; a partir de esa experiencia, realizamos ultrasonido como terapia antinflamatoria de rutina en el postoperatorio y obtuvimos recuperaciones más rápidas.

Los procedimientos auxiliares son importantes ya que ayudan a que nuestros resultados se vean aún mejor y en definitiva, no sólo suponen un beneficio para los pacientes, sino también para el cirujano.

Felicito al Dr. Antonio Fuente del Campo por su importante contribución, que será seguida por muchos colegas Latinoamericanos y del mundo.

\section{Respuesta al comentario del Dr. Guillermo Vázquez}

\section{Dr. Antonio Fuente del Campo}

El tema del envejecimiento así como los procedimientos para su restauración han acaparado gran parte de mi tiempo y atención en los últimos 20 años. En este artículo he querido referirme únicamente a la región frontal, para poder tratar el tema en profundidad mencionando los puntos que considero claves para obtener consistentemente buenos resultados y de aspecto natural.

Agradezco al doctor Guillermo Vázquez el amable comentario que hace de este artículo. El doctor Vázquez es un convencido de la importancia que tiene la restauración de los tejidos profundos para lograr un efecto de rejuvenecimiento global.

Sabemos que el envejecimiento implica pérdida de grasa subcutánea, tono y volumen muscular y pérdida de volumen óseo, vertical y anteroposterior. Estos cambios, en conjunto, alteran la relación entre el volumen facial y las dimensiones de la cubierta cutánea. Se pierde volumen facial y por lo tanto sobra piel que cae verticalmente afectada por la gravedad.
La restauración del rostro envejecido debe enfocarse a recuperar los volúmenes perdidos y corregir las alteraciones en la calidad y consistencia de la piel. Aunque la lipoinyección es de gran ayuda, en el momento actual no contamos con el recurso ideal para restaurar adecuadamente el volumen facial perdido. En estas circunstancias los procedimientos deben enfocarse a elevar y reubicar los tejidos blandos profundos, recuperar en lo posible el volumen perdido mediante lipoinyección y redistribuir la piel de acuerdo con las dimensiones vigentes de la cara. Estos procedimientos deben planearse para ser realizados a través del mínimo necesario de incisiones y evitar al máximo la secuela de las cicatrices. Todo esto auxiliado por las medidas pertinentes de Medicina Antienvejecimiento.

En próximos artículos nos referiremos a las aplicaciones de esta filosofía en el rejuvenecimiento de las mejillas, de la región centrofacial, de los parpados y del cuello. 